\title{
Simultaneous integrated boost intensity-modulated radiotherapy for locally advanced non-small cell lung cancer in Chinese population: A retrospective study
}

\author{
Yujin Xu' ${ }^{1,2}$, Xiao Zheng ${ }^{1,2}$, Xue $\mathrm{Bai}^{3}$, Pu Li ${ }^{3}$, Honglian Ma ${ }^{1,2}$, Jin Wang ${ }^{1,2}$, Xiao $\mathrm{Hu}^{1,2}$ \\ and Ming Chen ${ }^{1,2}$ \\ ${ }^{1}$ Department of Radiation Oncology, Zhejiang Cancer Hospital, Hangzhou, 310022, China \\ ${ }^{2}$ Zhejiang Provincial Key Laboratory of Radiation Oncology, Hangzhou, 310022, China \\ ${ }^{3}$ Department of Radiation Physics, Zhejiang Cancer Hospital, Hangzhou, 310022, China \\ Correspondence to: Ming Chen, email: chenming@zjcc.org.cn
}

Keywords: non-small cell lung cancer, integrated boost intensity-modulated radiotherapy, local control, overall survival

Received: March 03, $2017 \quad$ Accepted: March 31, $2017 \quad$ Published: April 13, 2017

Copyright: Xu et al. This is an open-access article distributed under the terms of the Creative Commons Attribution License 3.0 (CC BY 3.0 ), which permits unrestricted use, distribution, and reproduction in any medium, provided the original author and source are credited.

\section{ABSTRACT}

Objectives: To evaluate the clinical efficacy and toxicity of simultaneous integrated boost intensity-modulated radiotherapy (SIB-IMRT) for patients with locally advanced non-small cell lung cancer (NSCLC).

Results: All patients completed definitive radiotherapy and $74(85.1 \%)$ patients administrated platinum-based chemotherapy. The median radiation dose was $50.4 \mathrm{~Gy}$ to PTV and 64.4 Gy simultaneously to the PGTV. The overall response rate (ORR) was $57.5 \%(50 / 87)$. The median duration of follow up was 24.6 months. The 1, 2, 3-year local control rate was $79.0 \%, 66.1 \%$, and $60.5 \%$, respectively. The 1, 2, 3-year overall survival (OS) rate was $89.7 \%, 56.7 \%$, and $30.6 \%$, respectively. Subgroup analysis showed that the median OS in concurrent chemoradiation (CCRT) was much better than non-CCRT (35.7 vs. 26.4 months) (HR: 0.52, 95\% CI: $0.32-0.95, P=0.033$ ). Twenty-two (25.3\%) patients experienced acute grade 3 esophagitis and $10(11.5 \%)$ experienced acute grade $\geq 3$ radiation pneumonitis. There were $2(2.6 \%)$ late grade 3 pulmonary toxicity and no late grade $\geq 3$ esophageal toxicity was observed.

Materials and Methods: A total of 87 patients with locally advanced NSCLC who received SIB-IMRT from Jan. 2009 to Dec. 2012 in our hospital were retrospectively analyzed. Male accounted for $\mathbf{8 8 . 5} \%$, with a median age of 61 years old. The SIBIMRT plans were designed to deliver 50.4-64.0 Gy in 28-33 fractions (1.8-2.1 Gy/ fraction) to PTV while simultaneously delivering 60.0-74.3 Gy in 28-33 fractions (2.0-2.5 Gy/fraction) to PGTV.

Conclusions: SIB-IMRT, especially with concurrent chemotherapy, appears to be an effective and safe option to treat patients with locally advanced NSCLC. More prospective clinical studies should be warranted.

\section{INTRODUCTION}

About $30-40 \%$ cases of non-small cell lung cancer (NSCLC) belong to the locally advanced stage, which is not suitable for surgical resection. Radiation therapy, usually in combination with chemotherapy is the main treatment for these cases [1]. Prospective studies have shown that the two-year survival rate of stage III NSCLC after chemoradiation was between 22 to $33 \%$
[2]. Meanwhile, an improvement of radiation dose could significantly improve the local control and overall survival [3-5]. However, in order to avoid radioactive toxicity to normal tissue surrounded (e.g. radiation pneumonitis and esophagitis etc.), improving of target dose during conventional radiotherapy or three-dimensional conformal radiotherapy (3D-CRT) was usually not easy. Currently, some studies have suggested that the application of intensity-modulated radiotherapy (IMRT) in NSCLC 
treatment was superior to 3D-CRT in many ways, particularly in reducing the volume dose of normal tissue within radiation range while potentially improving the radiation dose that tumor delivered [6-8]. Yet in these studies regarding IMRT, the radiation dose targeting gross tumor volume and elective regional lymph nodes regions were the same, while technically the former should be higher than the dose delivered to subclinical volume. Simultaneous integrated boost intensity-modulated radiotherapy (SIB- IMRT) could simultaneously deliver a higher dose to the primary tumor volume and a relatively lower dose to the subclinical volume or elective regional lymph nodes regions. However, outcomes and safety for SIB-IMRT in locally advanced NSCLC remains unclear. In recent years, we have treated 87 cases of locally advanced NSCLC with SIB-IMRT technique, and now we perform a retrospective analysis to investigate the efficacy and safety of SIB-IMRT on locally advanced NSCLC.

\section{RESULTS}

\section{Patients characteristics}

A total of 87 patients enrolled in the study. Male accounted for $88.5 \%$, with a median age of 61 years old. The accompanied diseases before diagnosis included: $13(14.9 \%)$ of chronic obstructive pulmonary disease, 4 $(4.6 \%)$ of coronary heart disease, $5(5.7 \%)$ of diabetes, 18 of hypertension $(20.7 \%), 2(2.3 \%)$ of renal inadequacy, and $1(1.1 \%)$ of lower extremity thrombosis. Twenty-four $(27.6 \%)$ patients received concurrent chemoradiation (CCRT), while the remaining 63 (72.4\%) received sequential radiotherapy and chemotherapy or radiotherapy alone. The detailed clinical characteristics of 87 patients were summarized in Table 1.

\section{Radiotherapy completion}

All patients completed the prescribed radiotherapy. Most of patients (55 cases, 63.2\%) received 50.4Gy (in 28 fractions of $1.8 \mathrm{~Gy} /$ fraction) to planning target volume (PTV) and 64.4Gy (in 28 fractions at 2.3Gy/fraction) to the planning gross target volume (PGTV). Twenty-six $(30.0 \%$ ) patients received $56 \mathrm{~Gy}$ (in 28 fractions of $2.0 \mathrm{~Gy} /$ fraction) to PTV and 61.6Gy (in 28 fractions at $2.2 \mathrm{~Gy} /$ fraction) to the PGTV. Another 5 (5.7\%) received 54.0 Gy (in 30 fractions of $1.8 \mathrm{~Gy} /$ fraction) to PTV and 60.0 Gy (in 30 fractions at $2.0 \mathrm{~Gy} /$ fraction) to the PGTV. One (1.1\%) patient received 64.0 Gy (in 33 fractions at 1.94 Gy/fraction) to PTV and 74.3 Gy (in 33 fractions at 2.25 Gy/fraction) to the PGTV. The median mean lung dose (MLD) was 14.6 Gy (range 11.5-16.9 Gy), V20 was 24.7\% (range 19.9-31.8\%); esophageal V50, $37.1 \%$ (range 23.3-48.9\%); median mean heart dose, $25.4 \mathrm{~Gy}$ (range 2.6-28.8 Gy); and median spinal cord maximum dose, 37.4 Gy (range 28.6-45.2 Gy).

\section{Response evaluation}

One month after the completion of radiotherapy, chest and upper abdomen enhanced computed tomography (CT) was performed for the evaluation of efficacy. Of the 87 patients, overall response rate (ORR) was $57.5 \%$, with $13(14.9 \%)$ of complete response (CR), 37 (42.5\%) of partial response (PR), 34 (39.1\%) of stable disease (SD) and $3(3.4 \%)$ of progression disease (PD). Efficacy evaluation of different subgroups was shown in Table 2.

\section{Survival}

The median duration of follow up was 24.6 months, with a range of 3.8-40.5 months. Up to the newest followup, 58 in 87 patients have died, 29 surviving. Among all the 58 cases dead, 48 died of local recurrence or distant metastasis, three died of pulmonary infection after treatment, one case of pulmonary hemorrhage, and one died of accident. The other five patients had unknown reason. The one, two, three-year local control rate was 79.0\%, 66.1\% and $60.5 \%$, respectively (Figure 1A). The one, two, threeyear PFS rate was $54.0 \%, 30.6 \%$ and $26.8 \%$, respectively, with a median PFS of 14.5 months (Figure 1B). And the one, two, three-year OS rate was $89.7 \%, 56.7 \%$ and $30.6 \%$, respectively, with a median OS of 27.3 months (Figure 1C). In the subgroup analysis, the median OS in patients who received CCRT was much better than those who did not receive CCRT (35.7 vs. 26.4 months), indicating statistically significant difference between the two subgroups (HR: $0.52,95 \%$ CI: $0.32-0.95, P=0.033$ ) (Figure 2). While in the patients staged III A and III B, the median OS was 27.4 and 27.3 months, and there was no significant difference
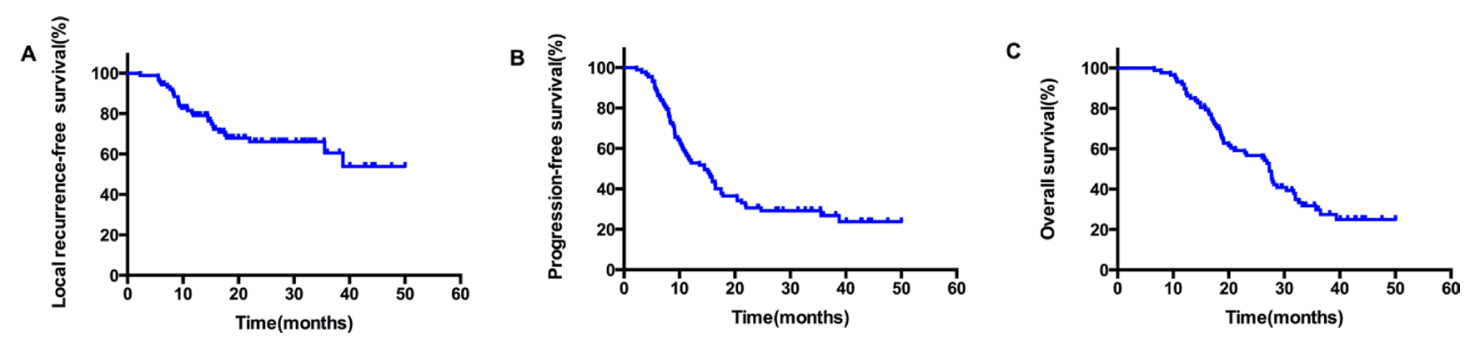

Figure 1: The survival curves in all patients who received SIB-IMRT, local recurrence-free survival (A); disease-free survival (B); Overall survival (C). 
Table 1: Demographics and clinicopathological characteristics of 87 patients

Factors

Number of cases

Percentage $(\%)$

Gender

Male

77

88.5

Female

10

11.5

Age (years)

Median (range)

$61(35-85)$

Smoking history

Yes

72

82.8

No

15

17.2

Clinical staging

III A

59.8

III B

Pathological type

$\mathrm{AC}$

SCC

NSCC NOS

Pathological grading

Well-differentiated

Moderately differentiated

4

24

Poorly differentiated

Undifferentiated

Unknown

Primary tumor type

Central

Peripheral

T stage

T1

$\mathrm{T} 2$

T3

T4

$\mathrm{N}$ stage

N0

N1

N2

N3

CCRT

Yes

No

IGTV volume $\left(\mathrm{cm}^{3}\right)$

Median (range)

PTV volume $\left(\mathrm{cm}^{3}\right)$

Median (range)

Abbreviations: AC, adenocarcinoma; SCC, squamous cell carcinoma; NSCC-NOS, non-small cell carcinoma-not otherwise specified; CCRT, concurrent chemoradiation; IGTV, internal gross tumor volume; PTV, planning tumor volume. 
Table 2: Efficacy evaluation of subgroup NSCLC patients [number of cases (\%)]

\begin{tabular}{lcccccc}
\hline Subgroup & CR & PR & SD & PD & ORR (\%) & P value \\
\hline Clinical staging & & & & & & \\
III A & $8(15.4)$ & $21(40.4)$ & $20(38.5)$ & $3(5.8)$ & 55.8 & 0.534 \\
III B & $5(14.3)$ & $16(45.7)$ & $14(40.0)$ & $0(0.0)$ & 60.0 & \\
Pathological type & & & & & \\
AC & $3(13.6)$ & $8(36.4)$ & $11(50.0)$ & $0(0.0)$ & 50.0 & \\
SCC & $6(11.5)$ & $26(50.0)$ & $18(34.6)$ & $2(3.8)$ & 61.5 & 0.317 \\
NSC NOS & $4(30.8)$ & $3(23.1)$ & $5(38.5)$ & $1(7.7)$ & 53.9 & \\
CCRT & & & & & & \\
Yes & $6(25.0)$ & $10(41.7)$ & $7(29.2)$ & $1(4.2)$ & 66.7 & 0.372 \\
No & $7(11.1)$ & $27(42.9)$ & $27(42.9)$ & $2(3.2)$ & 54.0 & \\
\hline
\end{tabular}

Abbreviations: AC, adenocarcinoma; SCC, squamous cell carcinoma; NSCC-NOS, non-small cell carcinoma-not otherwise specified; CCRT, concurrent chemoradiation; CR, complete response; PR, partial response; SD, stable disease; PD, progression disease; ORR, overall response rate.

(HR: $1.03,95 \%$ CI: $0.61-1.74, P=0.920$ ) (Figure 3). Likewise, when it came to pathological subgroups, we found there also was no significant difference among the different pathological types $(P=0.209)$ (Figure 4).

\section{Toxicity}

According to the Radiation Therapy Oncology Group (RTOG) toxicity score criteria, there were $65(74.7 \%)$ patients experienced acute radiation esophagitis, including $22(25.3 \%)$ were scored grade 3 radiation esophagitis and no patient occurred esophagitis above grade 4 . Among the 22 patients with grade 3 radiation esophagitis, $16(18.4 \%)$ had received CCRT, and the remaining $6(6.9 \%)$ patients had received sequential chemoradiation. Twenty-three $(26.4 \%)$ cases were developed acute radiation pneumonitis, including $10(11.5 \%)$ cases were grade 3 or above, with 8 $(9.2 \%)$ of grade 3 and 2 (2.3\%) of grade 4 . Among $8(9.2 \%)$ patients who developed grade 3 radiation pneumonitis, 6 $(6.9 \%)$ were patients who had received CCRT, with the dose of PTV 50.4-64.0 Gy, total lung V20 of 28.2-31.4\% and MLD of 14.3-16.7 Gy. And the two (2.3\%) patients of grade 4 acute radiation pneumonitis both occurred in stage IIIB patients who had suffered from severe ventilation abnormality before treatment (one case treated by consolidation chemotherapy after radiotherapy, the other treated by radiotherapy alone); and their dose of PTV was 60.0 Gy and 64.0 Gy, lung V20 was $27.6 \%$ and $30.7 \%$, and MLD was 15.4, 16.2 Gy, respectively. Late grade 3 pulmonary toxicity occurred in two (2.3\%) patients after one year of follow up, while no late esophagitis of grade 3 or above was observed. The acute and late toxicities in lung and esophagus were presented in detail (Table 3).

\section{Failure patterns}

The patterns of failure are detailed in Table 4. Of all 87 patients, a total of $63(72.4 \%)$ cases had disease progression, among which $28(32.2 \%)$ relapsed within the radiation field and seven $(8.0 \%)$ in the lymph node outside the radiation field. Another 28 patients developed distant metastasis, including 6 (6.9\%) intrapulmonary metastasis,

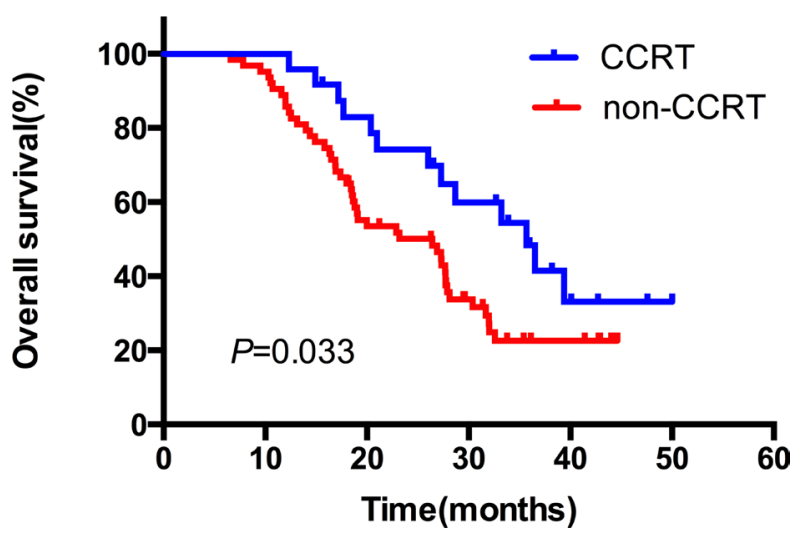

Figure 2: The comparative overall survival curves in patients who received CCRT or not. CCRT: concurrent chemoradiation. 
Table 3: Acute and late radiation toxicities

\begin{tabular}{lcccccccc}
\hline & \multicolumn{3}{c}{ Acute toxicity [n (\%)] } & \multicolumn{3}{c}{ Late toxicity [n (\%)] } \\
\cline { 2 - 8 } & Grade 1 & Grade 2 & Grade 3 & Grade 4 & Grade 1 & Grade 2 & Grade 3 & Grade 4 \\
\hline Esophagitis & $12(24.1)$ & $31(35.6)$ & $22(25.3)$ & $0(0.0)$ & $28(32.2)$ & $11(12.6)$ & $0(0.0)$ & $0(0.0)$ \\
Pneumonitis & $3(3.4)$ & $10(11.5)$ & $8(9.2)$ & $2(2.3)$ & $9(10.3)$ & $6(6.9)$ & $2(2.3)$ & $0(0.0)$ \\
\hline
\end{tabular}

Table 4: Failure patterns

\begin{tabular}{lcc}
\hline & $\boldsymbol{N}$ & $\mathbf{\%}$ \\
\hline Any failure & 63 & 72.4 \\
Locoregional failure & & 25.3 \\
PGTV failure & 22 & 6.9 \\
PTV failure & 6 & 8.0 \\
Out-field failure & 7 & 32.2 \\
Distant metastasis & 28 & 17.2 \\
Alive with failure & 15 & 16.1 \\
Alive with no failure & 14 & 55.2 \\
Death from treatment failure & 48 & 3.4 \\
Treatment-related death & 3 & 66.7 \\
Any death & 58 & \\
\hline
\end{tabular}

$9(10.3 \%)$ brain metastasis, $6(6.9 \%)$ bone metastasis, 3 (3.4\%) liver metastasis, 2 (2.3\%) pleural metastasis, 2 $(2.3 \%)$ adrenal metastasis.

\section{DISCUSSION}

For locally advanced NSCLC patients not suitable for completed resection, the local control and OS after radiotherapy or combined chemoradiation remains unsatisfactory. From early studies, the clinical control rate of NSCLC after conventional radiotherapy was about 50-70\%. However, when follow-up with fiberoptic bronchoscopy was used to evaluate the local control rate of NSCLC, it was found that the one-year local control rate of primary lung cancer was less than $20 \%$ [9]. The lack of local radiation dose in the primary tumor zone may be the main cause of failure for conventional radiotherapy. Kong [4] indicated that there was obvious dose-effect relationship in the radiation therapy of lung cancer, and that radiation dose escalation could directly improve local control rate. Therefore, to improve the local control rate and long-term survival even quality of life, radiation dose escalation was required. In our present study, we used the SIB-IMRT technology and the results showed a relatively better local control and median survival compared with previous published data $[1,10]$. Although only $27.6 \%$ patients received CCRT, the one, two, three-year local control rate was $79.0 \%, 66.1 \%$ and $60.5 \%$, respectively. We speculated that the relatively higher dose delivered to the gross tumor benefited the better local control, even OS. It should to be verified in the future perspective studies.

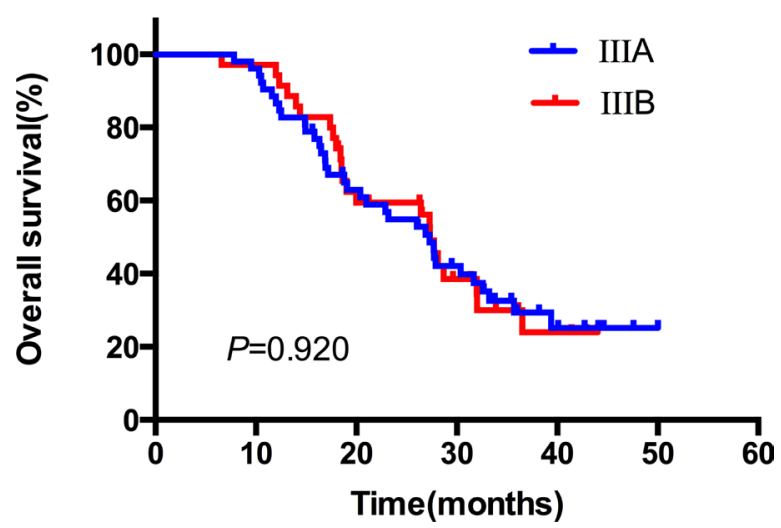

Figure 3: The comparative overall survival curves in patients with different clinical stages after SIB-IMRT. 
It has been reported that comparing with conventional IMRT technique, SIB-IMRT could increase GVT dosage by $22 \%$ with the same radiation dose on the surrounding normal tissue [11]. In our study, PGTV was delivered the total dose of 60.0-74.3 Gy with single dose of 2.0-2.3 Gy, five days per week. The median total dose was 64.4 Gy (in 28 fractions at 2.3 Gy/fraction). Previous study indicated that there was a positive association between radiation dose escalation and local control. When the tumor was delivered escalating dose of 63-69, 74-84 and 92-103 Gy, the control rates were $12 \%, 35 \%$ and $49 \%$, respectively [4]. RTOG 0617 trial was a phase III study comparing a dose of 60 Gy versus 74 Gy delivered concurrently with weekly paclitaxel and carboplatin \pm antibody against the epidermal growth factor receptor cetuximab in patients with locally advanced NSCLC. The results surprisingly showed that there was no OS improvement in the group randomized to the higher radiation dose. In fact, the survival in the highdose arm was worse [12]. Although the actual reasons for the unexpected results remains unclear, a competing explanation is that $74 \mathrm{~Gy}$ is too toxic when delivered by the photon-based radiation technologies used in current setting of RTOG 0617. Actually, 74 Gy may still be an inadequate dose for eliminating the tumor, just like the need for biological equivalent dose (BED) above of 100 Gy in early-stage NSCLC. Thus, safely escalating the radiation doses to the gross targets, while sparing the doses to the normal tissue surrounded may be critical to improving the outcome in NSCLC.

In the present study, a total of 82 patients received radiation with PGTV total dose of more than $6160 \mathrm{cGy}$ and single dose of more than 220cGy (BED was $62.63 \mathrm{~Gy}$ ) $(\alpha / \beta=10)$. Our long-term results showed that the one, two, three-year PFS rate was 54.0\%, 30.6\% and 26.8\%, respectively. The one, two, three-year OS rate was $89.7 \%, 56.7 \%$ and $30.6 \%$, respectively. The outcome made an improvement to OS and PFS compared with previous literature $[1,2,13,14]$. Meanwhile, in the subgroup analysis, we found that OS in patients treated with CCRT was significantly better than those who did not receive CCRT. On the other hand, we found that the incidence and severity of treatment-related toxicity, particularly late toxicity, was lower in our study than the previous reports $[15,16]$, and this was probably due to the following reasons: Prescription dose of PTV in our study was lower than in other studies (the median dose was 5040cGy, only enough for the irradiation of subclinical lesions and elective lymph nodes); Our study had more strict limit of the radiation on normal lung tissue, with lung V20 $<28 \%$ for most patients, MLD $<17 \mathrm{~Gy}$; proportion of the patients receiving standard CCRT was relatively low, for quite a few patients received sequential chemoradiation or radiotherapy alone instead, so that patient tolerance was better. As a combination of the above, we may conjecture that treatment intensity in our study could be increased still. So in our further research, standard CCRT or more radiation dose to PGTV should be applied under the premise of maintaining the intensity of radiotherapy, in order to achieve better local control and survival.

In terms of failure patterns, our results suggested most of the patients relapsed within the radiation field or distant metastasis. Only seven $(8.0 \%)$ relapsed in the lymph node outside the radiation field, indicating that the application of SIB-IMRT irradiation technique in the treatment of locally advanced NSCLC would not increase the recurrence rate of elective lymph node regions.

The present study had some limitation. Firstly, we did not use four-dimensional CT simulation, which could delineate more precise PGTV considering the tumor respiratory movement. Secondly, the size of enrolled population was small, which may cause the selection bias. Meanwhile, the treatment strategies of the patients were not uniform. Last but not least, positron emission tomography scan was rarely used before the treatment and during the period of follow-up, which means that some metastatic sites may have been missed and the location of failure in relation to the PGTV and PTV could not be accurately identified.

In conclusion, SIB-IMRT, especially with concurrent chemotherapy, appears to be an effective and safe option to

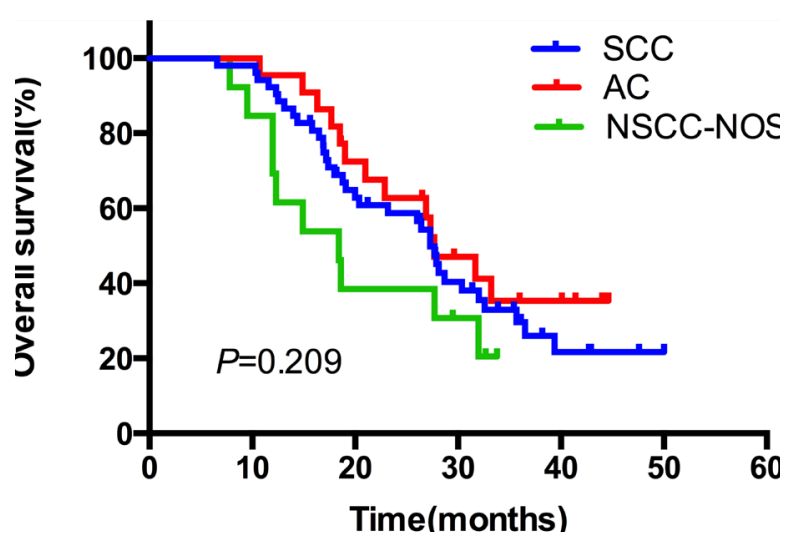

Figure 4: The comparative overall survival curves in patients with different pathological types after SIB-IMRT. 
treat patients with locally advanced NSCLC. However, our study was retrospective and only involved a few cases. The conclusion was still to be confirmed by further randomized controlled studies with larger sample sizes.

\section{MATERIALS AND METHODS}

\section{Patients selection}

All 87 cases involved were pathologically proved NSCLC patients in Zhejiang Cancer Hospital from January 2009 to December 2012. Inclusion criteria were as follows: age $\geq 18$ years; newly diagnosed cases of primary lung cancer; confirmed unsuitable to receive radical resection by surgeons or patient refusal; stage IIIA or IIIB according to the 7th edition of the American Joint Committee on Cancer (AJCC) staging manual for lung cancer; Eastern Cooperative Oncology Group (ECOG) performance status (PS) score of 0-2; Forced expiratory volume in the first second $(\mathrm{FEV} 1) \geq 1000 \mathrm{~cm}^{3}$, weight loss of less than $5 \%$ in the last three months; with measurable lesions by the response evaluation criteria in solid tumors (RECIST) 1.1 criteria; without other serious cardiopulmonary, hepatic or myeloid comorbidities. The study was approved by our hospital's review board. All participants gave written informed consent.

\section{Radiotherapy}

All patients took supine position and were fixed with thermoplastic mask, with arms crossed above the head. Enhanced CT scan was performed with large diameter spiral CT simulator (GE LightSpeed RT, USA) in quiet breathing conditions. Scanning range was from the upper edge of the second cervical vertebra to the lower edge of the second lumbar, and the thickness was $5 \mathrm{~mm}$. The scanned were transmitted to the Philips Pinnacle 9.2 three-dimensional treatment planning system (Philips Medical Systems, Bothell, WA). The gross target volume (GTV) was defined as any visible primary lesions, and all lymph nodes with a diameter $>$ $1 \mathrm{~cm}$ in short axis were included. The primary lesions were delineated in lung window (window width 850 $\mathrm{Hu}$, window level $-750 \mathrm{Hu}$ ) and positive lymph nodes and normal organs were determined in mediastinal window (window width $400 \mathrm{Hu}$, window level $20 \mathrm{Hu}$ ). The clinical target volume (CTV) was generated by expanding GTV a 6-8 $\mathrm{mm}$ margin and the high-risk lymph nodal regions and the ipsilateral hilar without extending into uninvolved organs. Another $0.6-1.0 \mathrm{~cm}$ margin was expanded to generate the PTV. PGTV was generated with an extension margin of $0.4-0.6 \mathrm{~cm}$ in the anterior-posterior (A-P) direction and left-right (L-R) direction, and $0.8-1.0 \mathrm{~cm}$ in the superior lobes, $1.0-1.5 \mathrm{~cm}$ in the mid or lower lobes in the cranialcaudal (C-C) direction. Prescription dose was defined as $95 \%$ of the receiving dose of PTV and PGTV, with the difference of internal target dose uniformity $<5 \%$, and internal target maximum dose point $\leq 110 \%$. Lung V20 (percentage of the total lung volume receiving 20 Gy or more radiation dose) was generally required to be less than $28 \%$ and was changed appropriately (not exceeding 34\%) according to patient's physical conditions and comprehensive treatment model. MLD $<17 \%$, maximum spinal cord dos $<45 \mathrm{~Gy}$; esophageal V $50<50 \%$, heart V40 $<50 \%$ were other demands to meet. Radiation was performed with linear accelerator $6 \mathrm{MV}-\mathrm{X}$. Of all 87 patients, prescription dose was PTV 50.4-64.0 Gy/28-33F, single dose 1.8-2.0 Gy; PGTV was 60.0-74.3 Gy/28-33F, with single dose of 2.0-2.3 Gy. The example of SIB-IMRT treatment plan was shown in Figure 5.
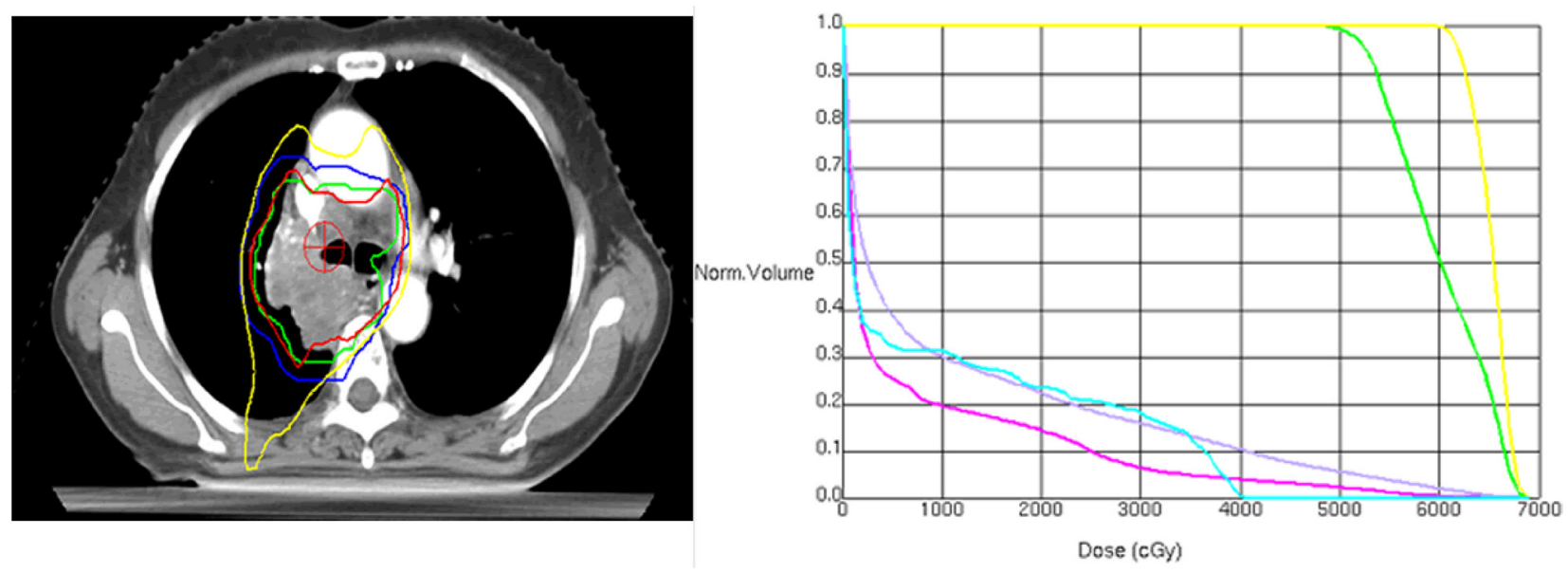

Figure 5: Isodose curves (left) and dose-volume histograms (right) of a simultaneously integrated boost intensity-modulated radiotherapy plan for a patients with locally advanced NSCLC (Left: green line represents the internal gross target volume (IGTV), blue line represents the planning target volume (PTV); Right: the yellow line outlines IGTV (61.6 Gy/28 fractions), and the green line area includes the PTV (50.4 Gy/28 fractions), red for heart, blue for spinal cord, gray for lung). 


\section{Chemotherapy and other therapy}

Among all 87 patients, 74 (85.1\%) patients administrated platinum-based chemotherapy, of which $24(27.6 \%)$ were CCRT and $50(57.5 \%)$ were sequential chemotherapy. Twenty-one patients received vinorelbine (25 mg/m2 intravenously, Days 1,8) and cisplatin (25 mg/m2 intravenously, Days 1-3); 28 cases of gemcitabine $(1000 \mathrm{mg} / \mathrm{m} 2$ intravenously, Days 1,8$)$ and cisplatin $(25 \mathrm{mg} / \mathrm{m} 2$ intravenously, Days 1-3); 22 cases of taxane-based (paclitaxel $135 \mathrm{mg} / \mathrm{m} 2$ or docetaxel 25 $\mathrm{mg} / \mathrm{m} 2$ intravenously, Day 1) chemotherapy combined with cisplatin ( $25 \mathrm{mg} / \mathrm{m} 2$ intravenously, Days 1-3), and another three cases of etoposide $(50 \mathrm{mg} / \mathrm{m} 2$ intravenously, Days 1-5) and cisplatin (50 $\mathrm{mg} / \mathrm{m} 2$ intravenously, Days 1,8) (all concurrent with radiotherapy). The number of chemotherapy cycles was ranged from one to six, with a median of four cycles. During treatment, seven of the 87 patients were given sodium glycididazole for synchronization sensitizing, and nine patients were given amifostine to avoid radioactive lung and esophageal injury. Two patients received concurrent thermotherapy and one received target therapy by taking erlotinib.

\section{Follow-up and efficacy evaluation}

The treatment response was evaluated within three months after radiotherapy referring to the Response Evaluation Criteria in Solid Tumors (version 1.1) [17]. After the first evaluation, follow-up should be performed every 3 months over the first 2 years and every 6 months thereafter. The medical test included physical examination, complete blood count, chest and upper abdomen CT, brain magnetic resonance imaging or $\mathrm{CT}$ scan, and bone scan (if necessary). On the other hand, the evaluation of toxicity in esophagus and lung was made according to the RTOG radiation injury grading standards.

\section{Statistical analysis}

We collected clinical and pathological data mainly on the following patient characteristics: gender, age, smoking history, clinical stage, pathological type, grading, primary tumor location, chemotherapy and target volume. A nonparametric Mann-Whitney $U$ test was used for comparisons among the efficacy of the groups. Failure patterns were defined as the first site of failure. Local recurrence included the primary tumor and regional lymph nodes. Survival duration was calculated from the first day of the treatment to that of the first occurrence of the considered event (local recurrence, disease progression, or death) or last follow-up time. Overall survival (OS), local recurrencefree survival (LRFS) and progression-free survival (PFS) rates were calculated by the Kaplan-Meier method and curves compared with log-rank tests. Cox proportional hazard models were used for the calculation of hazard ratio
(HR) of recurrence or death. $P<0.05$ was considered to be statistically significant. The reported confidence intervals (CI) are assumed to have a coverage probability of $95 \%$. All the analyses were performed using SPSS 21.0 (Statistical Package for Social Sciences, SPSS Inc., Chicago, IL, USA).

\section{CONFLICTS OF INTEREST}

The authors declare no conflicts of interest in this work.

\section{GRANT SUPPORT}

The present study was supported in part by two grants from Zhejiang medical science and technology foundation (grants No: 201480784; 2016146486).

\section{REFERENCES}

1. Curran WJ Jr, Paulus R, Langer CJ, Komaki R, Lee JS, Hauser S, Movsas B, Wasserman T, Rosenthal SA, Gore E, Machtay M, Sause W, Cox JD. Sequential vs. concurrent chemoradiation for stage III non-small cell lung cancer: randomized phase III trial RTOG 9410. J Natl Cancer Inst. 2011; 103:1452-60.

2. Belani CP, Choy H, Bonomi P, Scott C, Travis P, Haluschak J, Curran WJ Jr. Combined chemoradiotherapy regimens of paclitaxel and carboplatin for locally advanced non-small-cell lung cancer: a randomized phase II locally advanced multi-modality protocol. J Clin Oncol. 2005; 23:5883-91.

3. Sause W, Kolesar P, Taylor S IV, Johnson D, Livingston R, Komaki R, Emami B, Curran W Jr, Byhardt R, Dar AR, Turrisi A 3rd. Final results of phase III trial in regionally advanced unresectable non-small cell lung cancer: Radiation Therapy Oncology Group, Eastern Cooperative Oncology Group, and Southwest Oncology Group. Chest. 2000; 117:358-64.

4. Kong FM, Ten Haken RK, Schipper MJ, Sullivan MA, Chen M, Lopez C, Kalemkerian GP, Hayman JA. High-dose radiation improved local tumor control and overall survival in patients with inoperable/unresectable non-small-cell lung cancer: long-term results of a radiation dose escalation study. Int J Radiat Oncol Biol Phys. 2005; 63:324-33.

5. Wang L, Correa CR, Zhao L, Hayman J, Kalemkerian GP, Lyons S, Cease K, Brenner D, Kong FM. The effect of radiation dose and chemotherapy on overall survival in 237 patients with Stage III non-small-cell lung cancer. Int J Radiat Oncol Biol Phys. 2009; 73:1383-90.

6. Liu HH, Wang X, Dong L, Wu Q, Liao Z, Stevens CW, Guerrero TM, Komaki R, Cox JD, Mohan R. Feasibility of sparing lung and other thoracic structures with intensitymodulated radiotherapy for non-small-cell lung cancer. Int J Radiat Oncol Biol Phys. 2004; 58:1268-79.

7. Grills IS, Yan D, Martinez AA, Vicini FA, Wong JW, Kestin LL. Potential for reduced toxicity and dose 
escalation in the treatment of inoperable non-small-cell lung cancer: a comparison of intensity-modulated radiation therapy (IMRT), 3D conformal radiation, and elective nodal irradiation. Int J Radiat Oncol Biol Phys. 2003; 57:875-90.

8. Schwarz M, Alber M, Lebesque JV, Mijnheer BJ, Damen EM. Dose heterogeneity in the target volume and intensity-modulated radiotherapy to escalate the dose in the treatment of non-small-cell lung cancer. Int J Radiat Oncol Biol Phys. 2005; 62:561-70.

9. Arriagada R, Le Chevalier T, Quoix E, Ruffie P, de Cremoux H, Douillard JY, Tarayre M, Pignon JP, Laplanche A. ASTRO (American Society for Therapeutic Radiology and Oncology) plenary: Effect of chemotherapy on locally advanced non-small cell lung carcinoma: a randomized study of 353 patients. GETCB (Groupe d'Etude et Traitement des Cancers Bronchiques), FNCLCC (Féderation Nationale des Centres de Lutte contre le Cancer) and the CEBI trialists. Int J Radiat Oncol Biol Phys. 1991; 20:1183-90.

10. Fournel P, Robinet G, Thomas P, Souquet PJ, Léna H, Vergnenégre A, Delhoume JY, Le Treut J, Silvani JA, Dansin E, Bozonnat MC, Daurés JP, Mornex F, Pérol M, and Groupe Lyon-Saint-Etienne d'Oncologie ThoraciqueGroupe Français de Pneumo-Cancérologie. Randomized phase III trial of sequential chemoradiotherapy compared with concurrent chemoradiotherapy in locally advanced non-small-cell lung cancer: Groupe Lyon-Saint-Etienne d'Oncologie Thoracique-Groupe Français de PneumoCancérologie NPC 95-01 Study. J Clin Oncol. 2005; 23:5910-17.

11. Turner LM, Howard JA, Dehghanpour P, Barrett RD, Rebueno N, Palmer M, Vedam S, Klopp A, Komaki R, Welsh JW. Exploring the feasibility of dose escalation positron emission tomography-positive disease with intensity-modulated radiation therapy and the effects on normal tissue structures for thoracic malignancies. Med Dosim. 2011; 36:383-88.
12. Bradley JD, Paulus R, Komaki R, Masters G, Blumenschein G, Schild S, Bogart J, Hu C, Forster K, Magliocco A, Kavadi V, Garces YI, Narayan S, et al. Standard-dose versus high-dose conformal radiotherapy with concurrent and consolidation carboplatin plus paclitaxel with or without cetuximab for patients with stage IIIA or IIIB non-small-cell lung cancer (RTOG 0617): a randomised, two-by-two factorial phase 3 study. Lancet Oncol. 2015; 16:187-99.

13. Zatloukal P, Petruzelka L, Zemanova M, Havel L, Janku F, Judas L, Kubik A, Krepela E, Fiala P, Pecen L. Concurrent versus sequential chemoradiotherapy with cisplatin and vinorelbine in locally advanced non-small cell lung cancer: a randomized study. Lung Cancer. 2004; 46:87-98.

14. 14. Bradley J. A review of radiation dose escalation trials for non-small cell lung cancer within the Radiation Therapy Oncology Group. Semin Oncol. 2005; 32:S111-13.

15. Yom SS, Liao Z, Liu HH, Tucker SL, Hu CS, Wei X, Wang X, Wang S, Mohan R, Cox JD, Komaki R. Initial evaluation of treatment-related pneumonitis in advancedstage non-small-cell lung cancer patients treated with concurrent chemotherapy and intensity-modulated radiotherapy. Int J Radiat Oncol Biol Phys. 2007; 68:94-102.

16. Liao ZX, Komaki RR, Thames HD Jr, Liu HH, Tucker SL, Mohan R, Martel MK, Wei X, Yang K, Kim ES, Blumenschein G, Hong WK, Cox JD. Influence of technologic advances on outcomes in patients with unresectable, locally advanced non-small-cell lung cancer receiving concomitant chemoradiotherapy. Int J Radiat Oncol Biol Phys. 2010; 76:775-81.

17. Eisenhauer EA, Therasse P, Bogaerts J, Schwartz LH, Sargent D, Ford R, Dancey J, Arbuck S, Gwyther S, Mooney M, Rubinstein L, Shankar L, Dodd L, et al. New response evaluation criteria in solid tumours: revised RECIST guideline (version 1.1). European journal of cancer Oxford. 2009; 45:228-247. 